\title{
Monitoría de la FCF en el Desprendimiento Prematuro de Placenta Normoinserta (Abruptio Placentae)
}

Dres.: Quintero, C.A. Cobo, E., Cifuentes, R. y de Escobar, C*.

\section{INTRODUCCION}

El Abruptio Placentae (A. P.) ó Des. prendimiento prematuro de la placenta normoinserta, es el responsable de \pm $15 \%$ de las muertes perinatales (1). La literatura obstétrica siempre insiste en la operación cesárea como el método de elección temprano para finalizar el embarazo, cuando las condiciones materno-fetales lo indiquen, ó el parto vaginal, cuando se dan condiciones especiales. La frecuencia con la cual se presenta la entidad varía entre $0.4 \%$ y y $3.5 \%$ (2) de todos los partos

El manejo clínico de cada paciente se facilita si se tienen en cuenta, de una

I. Revisión de 4 años

II. Análisis de los cambios de la FCF

Departamento de Obstetricia y Ginecología Universidad del Valle, Cali. Colombia. parte, la clasificación de Page (3), que los diferencia en 4 grados de acuerdo a la severidad del cuadro, y de otra, la clasificación de Cobo y Cols. (4) que los diferencia en dos tipos de acuerdo a la actividad uterina. La primera clasificación es útil porque se basa en el estado materno $y$ fetal, permitiendo entonces decidir la forma y la intensidad del manejo terapéutico moderno. La segunda clasificación se hace necesaria porque diferencia aquellos casos sin hipertonía severa y con actividad uterina comparable a la normal, en los cuales se indica la administración de oxitocina si el feto está muerto, o el manejo conservador si el feto está vivo y el trazado de la frecuencia cardiaca fetal (F. C. F.) no muestra alteraciones severas.

El control del estado materno (T.A., hemorragia, presencia de fenómenos de 
coagulación, cambios del hematocrito, etc.), el estado fetal (F. C. F.) y equilibrio $A / B$, determinan que la operación cesárea ó la conducción del parto, sean las vías más elegibles para la culminación obstétrica del abruptio; sin embargo, consideramos de importancia efectuar un análisis de los diferentes patrones de FCF encontrados por nosotros con la monitoría electrónica y hacer algunos comentarios que nos hacen pensar en la multiplicidad de factores fisiopatológicos comprometidos en la entidad.

Desde el punto de vista de la monitoría fetal intraparto, o aún anteparto, en los casos en los cuales se ha llegado al diagnóstico de desprendimiento prematuro de placenta, no hay en la literatura una descripción que haga referencia a patrones únicos patognomónicos, o que correspondan a un determinado cuadro fisiopatológico responsable de la anoxia intrauterina a la cual se somete el feto en esta entidad, sino que toda la información se relaciona con la severidad del cuadro clínico, cambios en la FCFen términos de caída o aceleración, y que la operación cesárea es el método de elección cuando el feto está vivo (5.6. 7. 8). Ante esa situación hemos decidido estudiar los diferentes patrones de la FCF, haciendo énfasis en aquellos casos en los cuales las condiciones maternas, uterinas $y$ fetales permitan continuar realizando un control clínico del parto y obtener productos sanos sin necesidad de someter rutinariamente a la madre a una operación cesárea.

\section{Objetivos del trabajo}

1. Analizar durante un período de tiempo (4 años), los casos de abruptio placentae que tuvieron monitoría materno fetal $y$

2. Evaluar los cambios de la frecuencia cardíaca fetal, con el fin de estable- cer patrones de severidad dentro de los casos de A. P.

\section{Material y métodos}

Se hizo una revisión de todos los casos diagnosticados como A. P., atendidos en la sección de Obstetricia del Hospital Universitario del Valle, en el período comprendido entre Julio 1o. de 1975 y Junio 30 de 1979. Se utilizó este período $y$ no otro anterior, debido a que fue la época en la cual se inició la monitoría electrónica dentro de nuestro servicio. A todas las historias se les revisó el diagnóstico clínico y su comprobación una vez terminado el embarazo con el exámen de la placenta (grado de desprendimiento $\mathrm{y} / \mathrm{o}$ el peso del coágulo retroplacentario). Se hizo un análisis del estado materno al ingreso, tomando como referencia la T. A., la hemoglobina, el hematocrito, la presencia de hipertonía y el grado de la misma, la FCF y luego un seguimiento de la evolución de cada caso con monitoría electrónica y con evaluación del recién nacido por pediatra. Además, los casos de niños deprimidos que necesitaron cuidado intensivo se les hizo un seguimiento hasta su egreso.

Desde el punto de vista de la monitoría se utilizaron las técnicas descritas para monitoría fetal anteparto (métodos de registro externo) e intraparto (métodos de registro interno). Es de anotar que no se tomó el dato del estudio ácidobásico fetal o neonatal, por no haberse realizado o por ser incompletos los datos.

En lo que se refiere al análisis de la FCF se tomaron como parámetros importantes, siguiendo las normas establecidas por el grupo de Los Angeles $(9,10)$, los siguientes elementos:

a) Cambios en la línea de base: bradicardia o taquicardia. 
b) Cambios en la variabilidad: aumentada, disminuída o saltatoria (sinusoidal).

c) Cambios periódicos: desaceleraciones variables (leves, moderadas y severas) y desaceleraciones tardías.

d) Cambios periódicos combinados: desaceleraciones variables más componente tardío.

En cuanto a la clasificación del tipo de abruptio, seguimos la descrita según el grado de hipertonía (4). Tipo I sin hipertonía y actividad uterina normal o baja y, Tipo II con hipertonía severa (> $30 \mathrm{mmHg}$ ) y actividad uterina alta.

En nuestra revisión y análisis de datos se diferencian dos grupos de pacientes de acuerdo a la forma de terminación del embarazo, o sea, un grupo que terminó en operación cesárea y otro que fue al parto vaginal. Esta diferenciación, como se comentara mas adelante, no pretende establecer una norma de conducta definida en el sentido de indicar la operación cesárea o el parto vaginal como manejo ideal del abruptio placentae con feto vivo, sino por el contrario, fijar una serie de elementos de juicio que permitan evaluar individualmente cada caso $\mathrm{y}$ ejercer una acción pronta $\mathrm{y}$ objetiva que permita obtener un buen resultado materno fetal.

\section{Resultados}

Durante el período correspondiente entre el 1o. de Julio de 1975 y el 30 de Junio de 1979 se atendieron en el servicio de partos de la Unidad de Obstetricia del Hospital Universitario del Valle un total de 29.562 partos de los cuales 667 corespondieron al diagnóstico clínico de abruptio placentae $(2.3 \%)$. Además se realizaron 3.010 monitorías intraparto $(10.2 \%)$ y un total de cesáreas de 4.053 $(13.7 \%)$, de las cuales 387 fueron en pacientes con el diagnóstico de la enti-
Cuadro No. 1

HOSPITAL UNIVERSITARIO DEL VALLE

DEPARTAMENTO DE OBSTETRICIA Y GINECOLOGIA

Julio 1o. de 1975 - Junio 30 de 1979

\begin{tabular}{|l|r|r|}
\hline & \multicolumn{1}{|c|}{$n$} & \multicolumn{1}{c|}{$\%$} \\
\hline PARTOS & 29562 & - \\
MONITORIAS & 3010 & 10.2 \\
CESAREAS & 4053 & 13.7 \\
ABRUPTIO & & \\
PLACENTAE & 667 & 2.3 \\
CESAREAS CON & 387 & 5.8 \\
ABRUPTIO & 38 & \\
\hline
\end{tabular}

No. de partos atendidos en el período comprendido entre julio 10 . de 1975 y junio 30 de 1979 y la distribución porcentual de pacientes con diagnóstico de abruptio placentae, monitorías realizadas intra y anteparto, cesáreas en general y cesáreas en pacientes con diagnóstico de abruptio placentae.

dad que nos ocupa $(58 \%)$. (Cuadro No 1). La distribución de casos atendidos por períodos correspondientes desde Julio 1o. de 1975 a Junio 30 de 1979 (Cuadro No. 2) y el total de ellos demuestra que el A.P. se presentó en una proporción de uno por cada 44 partos, con una distribución de prevalencia de la entidad más o menos constante.

Estudiamos 65 casos que fueron los que llenaron el requisito indispensable de tener comprobación postparto o postcesárea del diagnóstico, y descartamos aquellos que aunque clínicamente tuvieron el diagnóstico no aparecía el resultado final del estudio de la placenta. De estos casos, 27 terminaron en parto vaginal $(41.6 \%)$ y 38 en operación cesárea $(58.4 \%)$. (Cuadro No. 3 ).

Al analizar las edades de las pacientes así como la paridad, encontramos que el 
Cuadro No. 2

\section{TOTAL DE PARTOS Y ABRUPTIOS EN PACIENTES ATENDIDAS}

\begin{tabular}{|c|c|r|r|}
\hline Años & \multirow{2}{*}{$\begin{array}{c}\text { No. de } \\
\text { partos }\end{array}$} & \multicolumn{2}{|c|}{ Abruptios } \\
\cline { 3 - 4 } $\begin{array}{c}1975 \\
\text { (Desde Julio 1o.) }\end{array}$ & 3515 & 87 & 2.5 \\
\hline 1976 & 8198 & 140 & 1.7 \\
\hline 1977 & 7016 & 189 & 2.7 \\
\hline 1978 & 7079 & 164 & 2.3 \\
\hline $\begin{array}{c}1979 \\
\text { (Hasta Junio 30) }\end{array}$ & 3754 & 87 & 2.3 \\
\hline TOTAL & 29562 & 667 & 2.3 \\
\hline
\end{tabular}

(1:44 Partos)

Distribución del número de partos y abruptios en pacientes atendidas entre el 10 . de julio de 1975 y el 30 de junio de 1979 en el Hospital Universitario del Valle.

Cuadro No. 3

\begin{tabular}{|l|c|c|}
\hline $\begin{array}{c}\text { Terminación del } \\
\text { Embarazo }\end{array}$ & $\mathrm{n}$ & $\%$ \\
\hline PARTOS & 27 & 41.6 \\
\hline CESAREAS & 38 & 58.4 \\
\hline TOTAL & 65 & 100.0 \\
\hline
\end{tabular}

Modo de terminación del embarazo. Nótese un porcentaje mayor del número de cesáreas.

$66.2 \%$ eran mujeres con menos de 30 años, con un rango de edades entre 25 y 44 años; también fue notoria la proporción de nulíparas $35.4 \%$.

Desde el punto de vista del estadio clínico de las pacientes al ingreso, es importante realzar los siguientes puntos:
1. Ninquna de estas pacientes llegaron al hospital en estado crítico, desde el punto de vista de choque o anemia aguda.

2. Hubo una asociación con enfermedad hipertensión y/o toxemia del $43.1 \%$. también como evento ligado al anterior, encontramos una proporción de $R$. N. de bajo peso para la edad gestacional de $35 \%$.

En la mayoría de los casos se hizo monitoría intraparto $(87.7 \%)$ y en el resto fue monitoría anteparto $(12.3 \%)$. En estos últimos existía patología asociada tal como toxemia, o algún sangrado con o sin hipertonía y se había descartado la presencia de otra entidad.

Vale la pena anotar que un caso correspondió a un desprendimiento de placenta provocado por una inducción médica del parto en una paciente con diagnóstico de ruptura prematura de membranas, quien presentó una polisistolia y posteriormente un cuadro de sufrimiento fetal agudo (Figura No. 1, panel A y B).

En relación a la evaluación inmediata del recién nacido tuvimos que tomar el dato del indice de Apgar a los 5' (Cuadro No. 4), debido a que el que se tomó en el primer minuto era incompleto $y$ vemos cómo el $40 \%$ de los fetos nacieron o estuvieron deprimidos a los 5'. Debe anotarse que de estos niños deprimidos murieron en el período neonatal 5: 1 postparto y 4 postcesárea.

En lo que a la Mortalidad Perinatal se refiere, hubo en el grupo analizado 8 muertes perinatales distribuídas asi: 3 mortinatos y 5 muertes neonatales en los primeros 7 días de vida (Cuadro No. 5).

Siguiendo nuestra clasificación en tipos I y II, de acuerdo al grado de hipertonía (Figura 2, panel $A, B$ ) encontra$\operatorname{mos} 2 y$ casos $(47.7 \%)$ sin hipertonía 


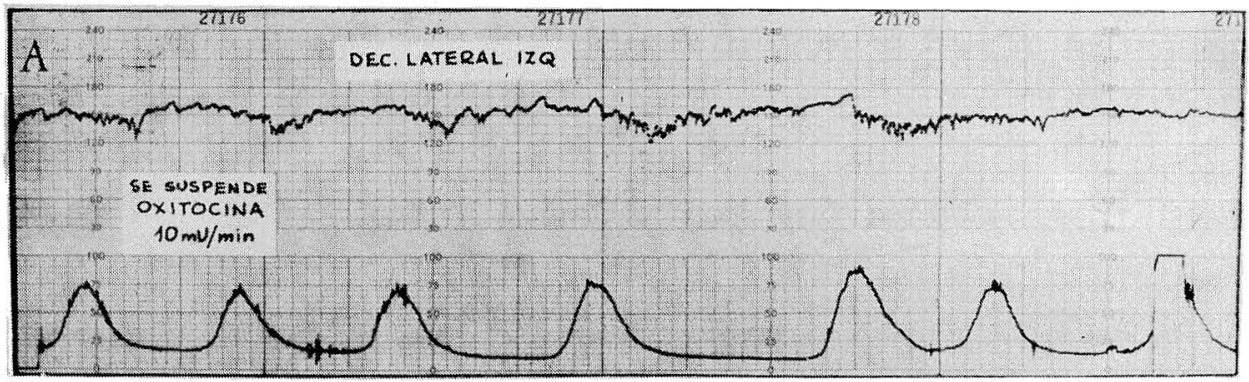

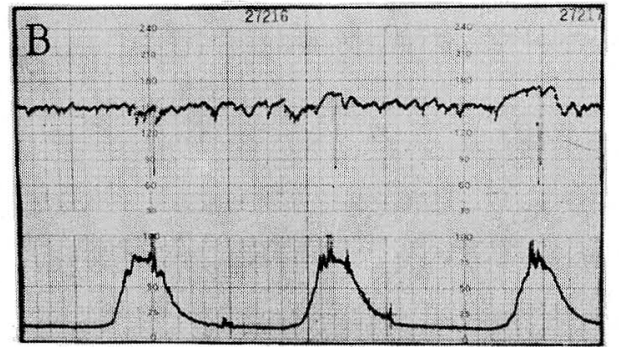

Fig. No. 1. Patrón de FCF encontrado en una paciente con ruptura prematura de membranas y a quien se le indujo el parto. Panel A. Nótese las desaceleraciones tardías las cuales desaparecen al disminuir la actividad uterina. Panel B.

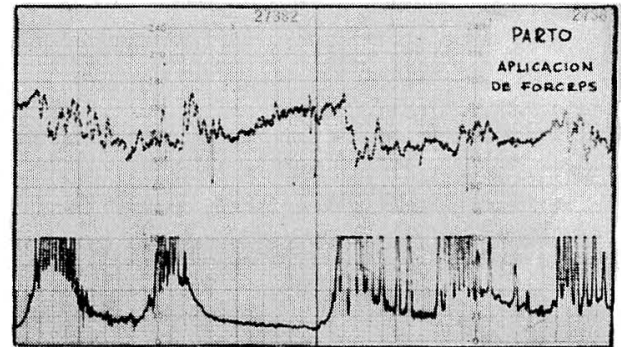

Actividad uterina y FCF normales en la primera parte y cambios de la FCF en el expulsivo (Desaceleraciones con disminución de la línea de la base) el cual fue instrumentado. Se encontró un desprendimiento del $20 \%$.

\section{Cuadro No. 4}

\begin{tabular}{|l|c|c|c|c|c|c|}
\hline Ind. de Apgar (5') & Parto & $\%$ & Cesárea & $\%$ & Total & $\%$ \\
\hline 7 Y MAS & 18 & 66.6 & 18 & 47.3 & 36 & 55.4 \\
\hline 6 Y MENOS & 7 & 25.9 & 19 & 50.0 & 26 & 40.0 \\
\hline SIN DATO & 2 & 7.4 & 1 & 2.6 & 3 & 4.6 \\
\hline TOTAL & 27 & & 38 & & 65 & 100 \\
\hline
\end{tabular}

Distribución del índice de Apgar a los 5 minutos del nacimiento. Obsérvese que el $40 \%$ tuvieron un índice menor de 7 (deprimidos).

y $36(52.3 \%)$ con hipertonía severa. Los casos de tipo I terminaron en el $55.2 \%$ en parto vaginal $y$ en el $44.8 \%$ en operación cesárea. En los casos de tipo II fue necesario realizar operación cesárea en el $69.4 \%$ (Cuadro 6). De 


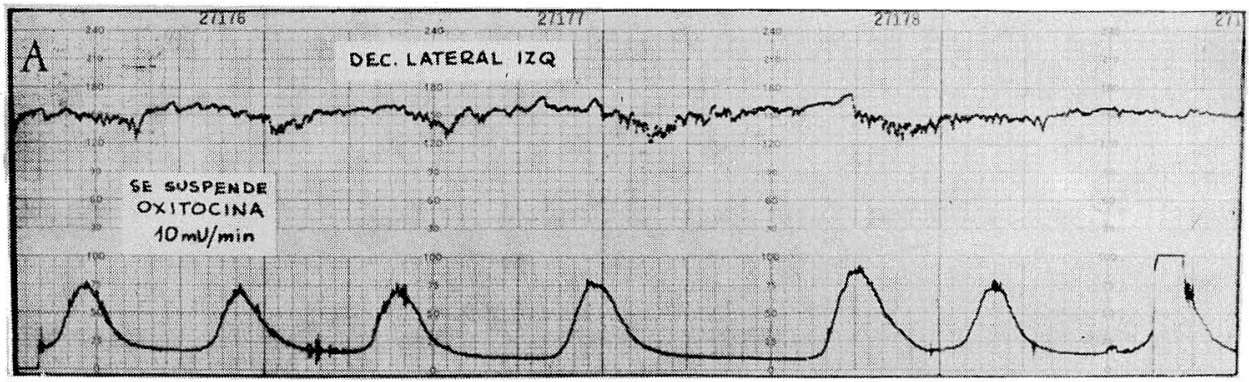

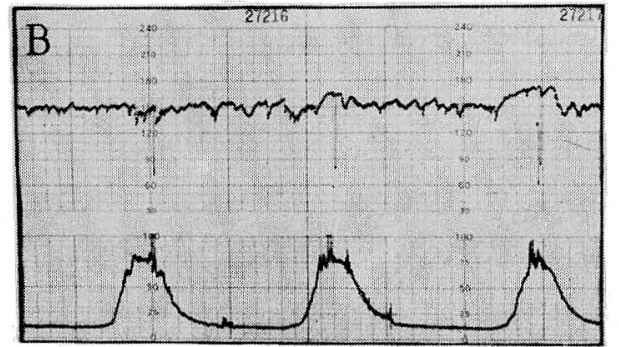

Fig. No. 1. Patrón de FCF encontrado en una paciente con ruptura prematura de membranas y a quien se le indujo el parto. Panel A. Nótese las desaceleraciones tardías las cuales desaparecen al disminuir la actividad uterina. Panel B.

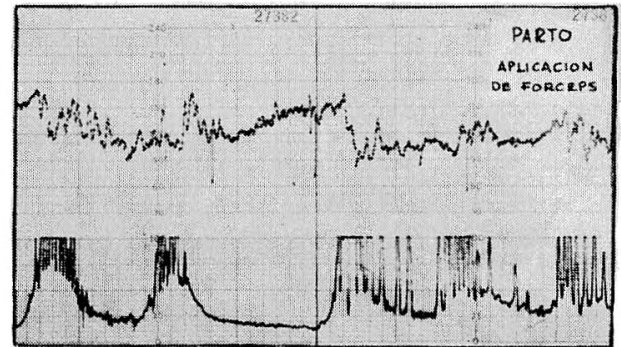

Actividad uterina y FCF normales en la primera parte y cambios de la FCF en el expulsivo (Desaceleraciones con disminución de la línea de la base) el cual fue instrumentado. Se encontró un desprendimiento del $20 \%$.

\section{Cuadro No. 4}

\begin{tabular}{|l|c|c|c|c|c|c|}
\hline Ind. de Apgar (5') & Parto & $\%$ & Cesárea & $\%$ & Total & $\%$ \\
\hline 7 Y MAS & 18 & 66.6 & 18 & 47.3 & 36 & 55.4 \\
\hline 6 Y MENOS & 7 & 25.9 & 19 & 50.0 & 26 & 40.0 \\
\hline SIN DATO & 2 & 7.4 & 1 & 2.6 & 3 & 4.6 \\
\hline TOTAL & 27 & & 38 & & 65 & 100 \\
\hline
\end{tabular}

Distribución del índice de Apgar a los 5 minutos del nacimiento. Obsérvese que el $40 \%$ tuvieron un índice menor de 7 (deprimidos).

y $36(52.3 \%)$ con hipertonía severa. Los casos de tipo I terminaron en el $55.2 \%$ en parto vaginal $y$ en el $44.8 \%$ en operación cesárea. En los casos de tipo II fue necesario realizar operación cesárea en el $69.4 \%$ (Cuadro 6). De 
los 5 casos de muertes neonatales descritos atrás, 4 correspondieron a cuadros de hipertonía severa y el otro a un caso sin hipertonía pero con al teraciones de la línea de base y con desaceleraciones tardías severas.

Cuadro No. 5

\begin{tabular}{|c|c|c|c|c|c|c|}
\hline \multirow{2}{*}{$\begin{array}{l}\text { Mortalidad } \\
\text { perinatal }\end{array}$} & \multicolumn{2}{|c|}{$\begin{array}{c}(27) \\
\text { Partos }\end{array}$} & \multicolumn{2}{|c|}{$\begin{array}{c}(38) \\
\text { Cesáreas }\end{array}$} & \multicolumn{2}{|c|}{ Total } \\
\hline & $\mathrm{n}$ & $\%$ & $\mathbf{n}$ & $\%$ & $\mathrm{n}$ & $0 / 0$ \\
\hline MORTINATOS & 1 & 3.7 & 2 & 5.3 & 3 & 4.65 \\
\hline $\begin{array}{l}\text { M. NEONATALES } \\
\text { 1os. } 7 \text { DIAS }\end{array}$ & 1 & 3.7 & 4 & 10.5 & 5 & 7.65 \\
\hline TOTAL & 2 & 7.4 & 6 & 15.8 & 8 & 12.3 \\
\hline
\end{tabular}

Mortalidad perinatal en la muestra seleccionada.

$$
\text { TIPOIA }
$$
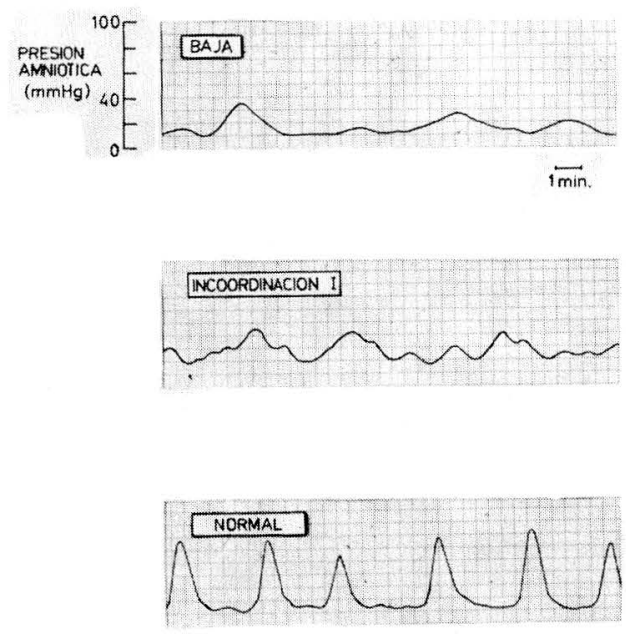

TIPO II B
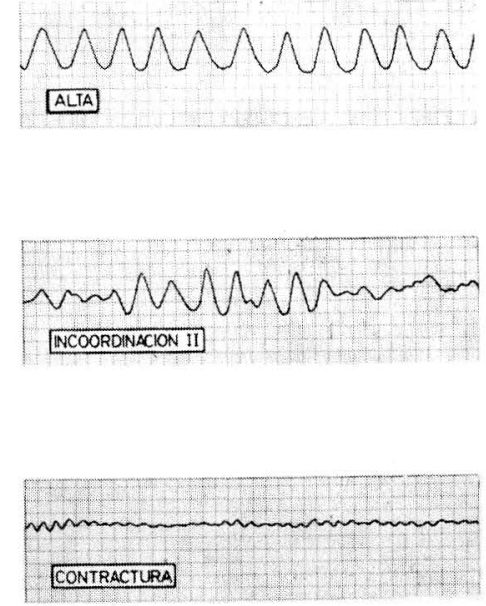

Fig. No. 2. Clasificación de los tipos de abruptio de acuerdo al grado de hipertonía (Cobo y Col.) Tipo I sin hipertonía. Tipo II hipertonía $>$ de $30 \mathrm{mmHg}$. 
Cuadro No. 6

\begin{tabular}{|l|c|c|c|c|c|c|}
\hline \multicolumn{1}{|c|}{$\begin{array}{c}\text { Grado de } \\
\text { Hipertonia }\end{array}$} & \multirow{2}{*}{$n$} & $\%$ & \multicolumn{2}{|c|}{ Parto } & \multicolumn{2}{|c|}{ Cesárea } \\
\cline { 4 - 7 } & & & $n$ & $\%$ & $n$ & $\%$ \\
\hline $\begin{array}{l}\text { HIPERTONIA } \\
30 \mathrm{~mm} \mathrm{Hg} \mathrm{o} \\
\text { A. Tipo I }\end{array}$ & 36 & 55.4 & 11 & 30.6 & 25 & 69.4 \\
\hline $\begin{array}{l}\text { SIN HIPERTONIA } \\
\text { A. Tipo II }\end{array}$ & 29 & 44.6 & 16 & 55.2 & 13 & 44.8 \\
\hline
\end{tabular}

Tipo de abruptio según la clasificación del grado de hipertonía. Nótese que más o menos

La línea de base de la FCF mostró las siguientes alteraciones: Disminución y/o pérdida de la variabilidad, taquicardia bradicardia y patrón sinusoidal. La proporción de dichos cambios en los casos estudiados fue la siguiente: (gráfico No. 1).

\section{GRAFICO No. 1}

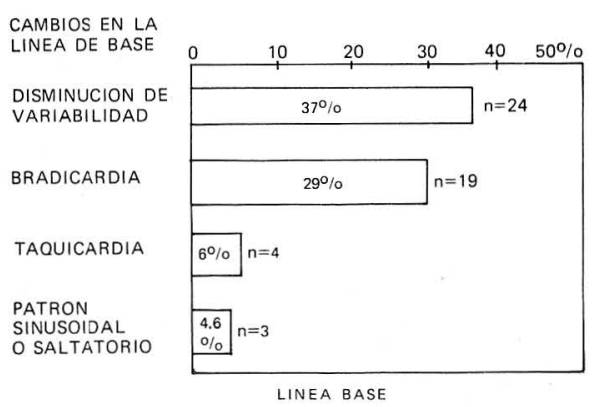

Cambios en la Línea de la Base de la FCF. Se observa una mayor porporción de disminución de la variabilidad y bradicardia.

a) Pérdida $y / o$ disminución de variabilidad

b) Bradicardia la mitad de los casos no tenían hipertonía o era leve.

c) Taquicardia

d) Patrón sinusoidal, o Saltatorio

En un caso que tuvo parto vaginal con feto muerto posiblemente hubo un error en la apreciación del trazado de la FCF (Figura No. 3), en el sentido de que se tuvo en cuenta la tendencia a un patrón saltatorio, se asimiló a una línea de trazado aparentemente normal y se suspendió la monitoría 4 horas antes del parto sin poder evaluar los cambios que posteriormente concluyeron en un mortinato.

Dos casos de muertes fetales que ingresaron con feto vivo correspondieron a un caso con bradicardia y patrón sinusoidal o saltatorio que posiblemente correspondía al registro de la frecuencia cardiaca materna, no había hipertonía uterina y el feto presentó signos de rigidez al nacer, 30 minutos después de haber terminado el registro, tiempo durante el cual se preparó la paciente para una operación cesárea (Figura No. 4) y otro caso de hipertensión con toxemia sobreagregada cuyo registro anteparto mostró una línea de base recta con ausencia de movimientos fetales espontáneos (Figura No. 5) que se llevó a 

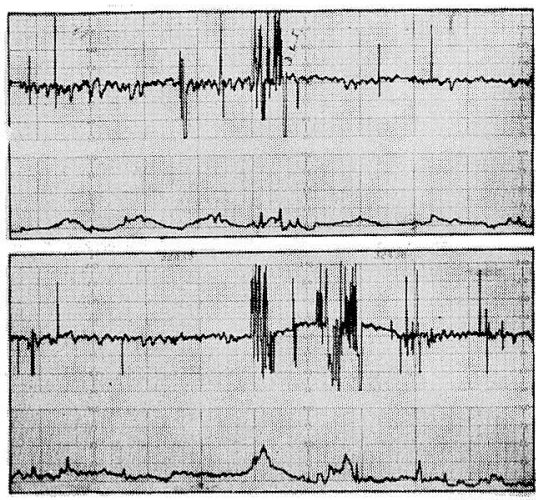

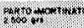

orenevapient

Fig. No. 3. FCF con tendencia a un ritmo saltatorio. Cuatro horas más tarde sin monitoría se obtuvo un feto muerto-Abruptio Tipo I.

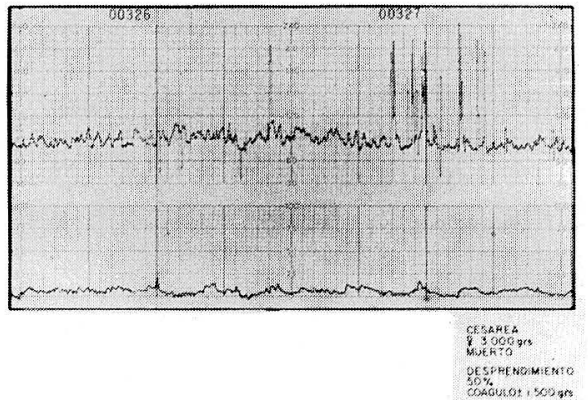

Fig. No. 4. FCF posiblemente materna, Mortinato con signos de rigidez.

cirugía 8 horas más tarde, obteniéndose un feto muerto. Vale la pena mencionar que los casos que tuvieron disminución de variabilidad y que fueron llevados a operación cesárea fueron los que estuvieron más comprometidos con el resultado fetal al nacimiento (Apgar menor de 6) o con mortalidad neonatal (Figura No. 6). Los casos de patrón sinusoidal o saltatorio se asociaron a mortalidad fetal (1 caso) y a morbimortalidad neonatal inmediata (2 casos).

Los cambios periódicos observados fueron los siguientes: (gráfico No. 2)
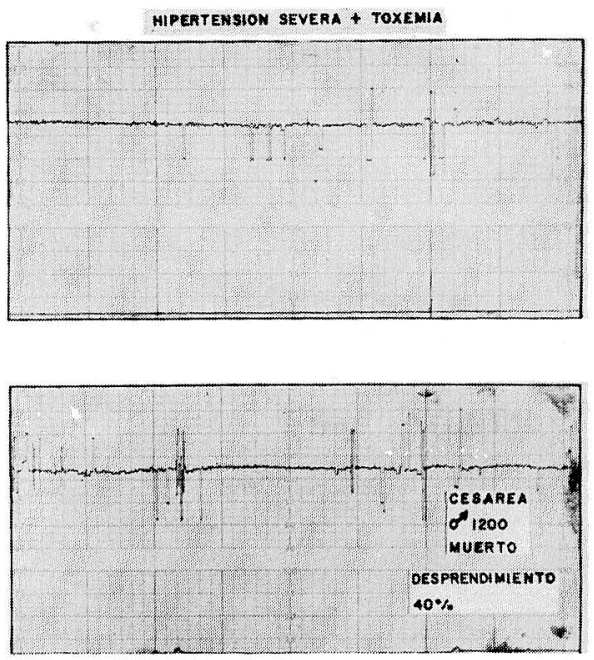

Fig. No. 5. Monitoría anteparto. Nótese línea de base casi plana sin movimientos fetales (NST no reactiva). Operación cesárea 8 horas más tarde; se obtuvo un feto muerto.

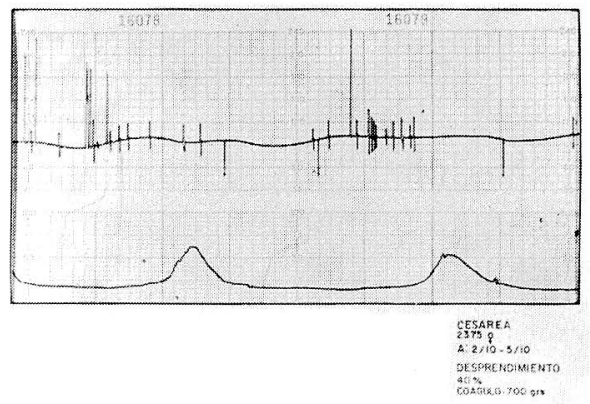

Fig. No. 6. Nótese la bradicardia (Línea de base $100 \mathrm{~L} / \mathrm{min}$ ), la pérdida de variabilidad y las desaceleraciones tardías. Recién nacido deprimido severamente. 
a) Desaceleraciones tardías

b) Desaceleraciones variables severas

c) Desaceleraciones variables moderadas
$\%$

50.8

20.0

17.0 g) Aceleraciones variables

GRAFICO No. 2

CAMBIOS PERIODICOS

DESACELERACION TARDIA
DESACELERACION VARIABLE
SEVERA

DESACELERACION VARIABLE + COMPONENTE TARDIO

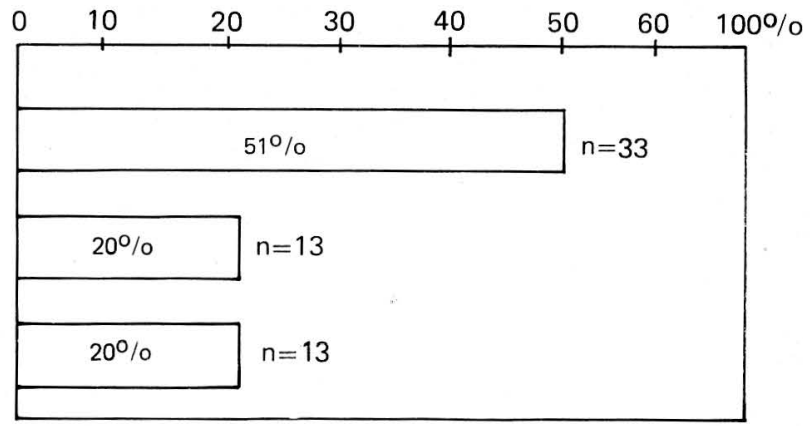

Cambios periódicos de la FCF. Las desaceleraciones tardías fueron los cambios más frecuentes.

La presencia de cambios periódicos fue el elemento más destacado dentro de los casos como se observa en el Gráfico No. 2. Hubo un predominio de signos de insuficiencia úteroplacen taria (desaceleraciones tardías a través de los dos períodos del parto; sin embargo, aclaramos que este tipo de desaceleraciones en los casos que se llevaron al parto vaginal fueron ocasionales (Figura No. 7) y su presencia desapareció con medidas terapéuticas tales como: cambios de posición materna, oxígeno, supresión de infusiones de oxitocina, etc. (Figura No. 8), pero en casi todos ( 6 casos) se hicieron presentes en el segundo período del parto. Los casos de desaceleraciones tardías que no se modificaron durante 30 minutos fueron llevados a operación cesárea
(Figura No. 9). El patrón de FCF cuyos cambios son atribuíbles a compresión de cordón no fueron tan manifiestos aunque si es importante hacer notar la presencia de un buen número de desaceleraciones variables moderadas y severas en el período expulsivo (Figura No. 10). Los casos que presentaron el patrón de FCF de desaceleraciones variables más componente tardío y pérdida o disminución de la variabilidad se asociaron con una alta proporción de R. N. deprimidos (5 de 7). (Figura No. 11).

\section{Comentarios}

Se ha hecho una revisión de una serie de casos de desprendimiento prematuro de placenta con el fin de analizar la utilidad de la monitoría electrónica en el 

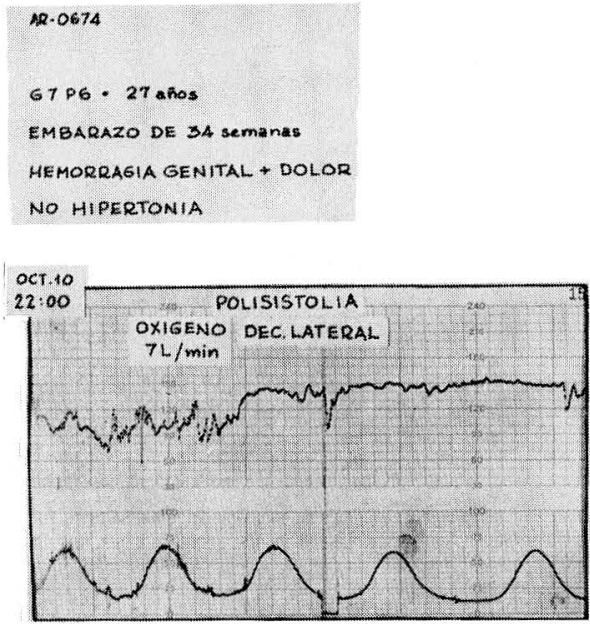

Fig. No. 7. Cambios periódicos de la FCF que se modificaron con medidas terapéuticas.
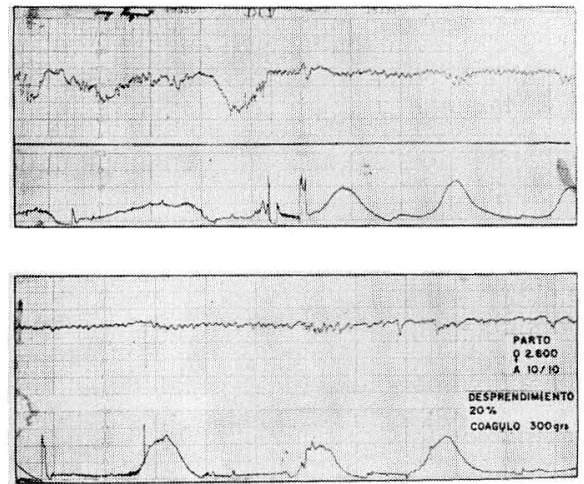

Fig. No. 8. Desaceleraciones tardías ocasionales con registro de FCF normal durante el expulsivo.

seguimiento $\mathrm{y}$ la conducción de estos casos con el objeto de asignar algunos parámetros de conducta oportuna y reconocer las variables que se presentan desde el punto de vista biofísico a través de análisis de los diferentes patrones de
FCF y haciendo énfasis en que la presencia de ciertos elementos de juicio podrían ser importantes en el resultado fetal y en el pronóstico del recién nacido.

1. Consideramos que después de una adecuada valoración del grado clínico del abruptio (Page) y el tipo de patrón contractil (Cobo) es obligatoria la monitorización de la actividad uterina y la frecuencia cardiaca fetal, para definir objetivamente una conducta.

Según la descripción original de Cobo, aproximadamente la mitad de los casos de abruptio placentae no presentan el patrón de actividad uterina descrito clásicamente como hipertonía esencial. Este hallazgo se ha confirmado en este trabajo en el cual el $45 \%$ de los casos cursaron sin hipertonía o con hipertonía moderada.

2. Es fundamerital reconocer los patrones de FCF que implican un compromiso fetal severo y el momento en el 


$$
\text { T. } 0537
$$

PRIMIGRAVIDA $\cdot 24$ años

EMBARAZO DE TERMINO

PQECLAMPSIA SEVERA

HIPERTONIA UTERINA $=30 \mathrm{mmH} / \mathrm{S}$

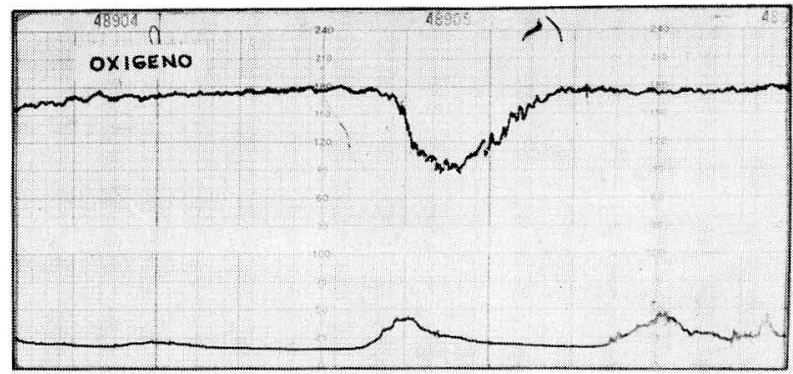

ABRUPTIO PLACENTAE TIPO II
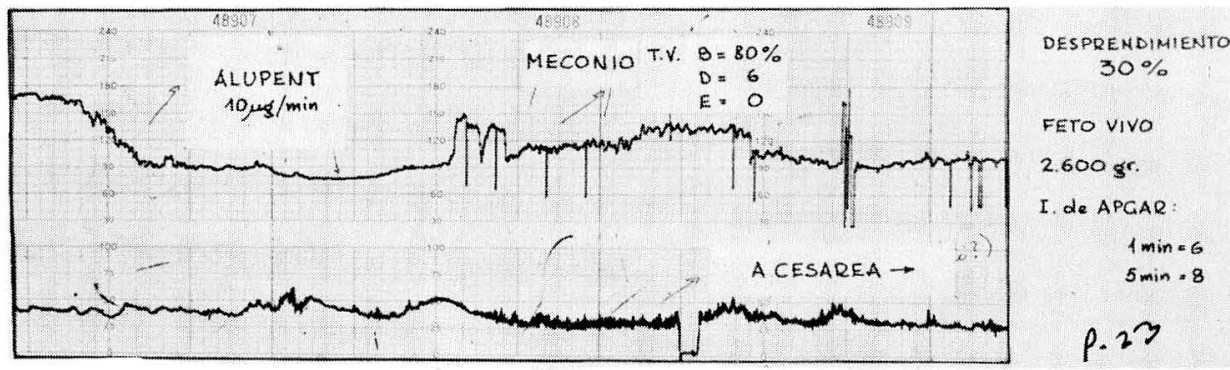

Fig. No. 9. Cambios periódicos que no se modificaron con medidas terapéuticas y que se

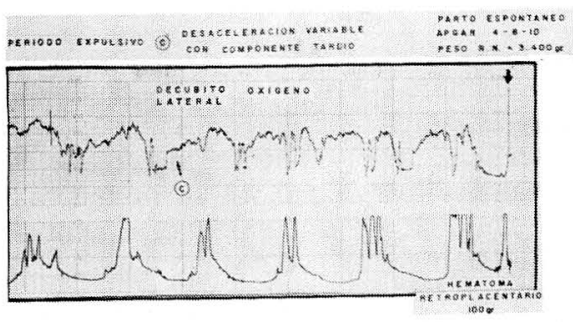

Fig. No. 10. Desaceleraciones variables durante el período expulsivo.

cual aparecen estos cambios ya que ellos parecen depender de las condiciones de reserva fetal, así como de la severidad y la duración de la anoxia $(11,12)$. Posiblemente esto explique por qué no se observan desaceleraciones tardías en to- acompañaron de signos de empeoramiento de la FCF.

dos los casos y sugiere que ellas sólo aparecerían en aquellos casos con baja reserva fetal $\mathrm{y} / \mathrm{o}$ anoxia severa o prolongada (13).

Lo anterior sumado al hecho de que durante las contracciones disminuye el aporte de sangre y oxígeno al feto y más aún en condiciones tales como toxemia, hipertensión, hipotensión materna, etc. donde hay un compromiso mayor de la Unidad Feto-Placenta, explican el hecho de que los efectos sobre el feto sean más pronunciados y lo lleven hacia el deterioro y la muerte intrauterina o neonatal temprana.

3. En el mismo orden de ideas de lo anteriormente expuesto, hacemos énfasis en que hay ciertos elementos que permi- 


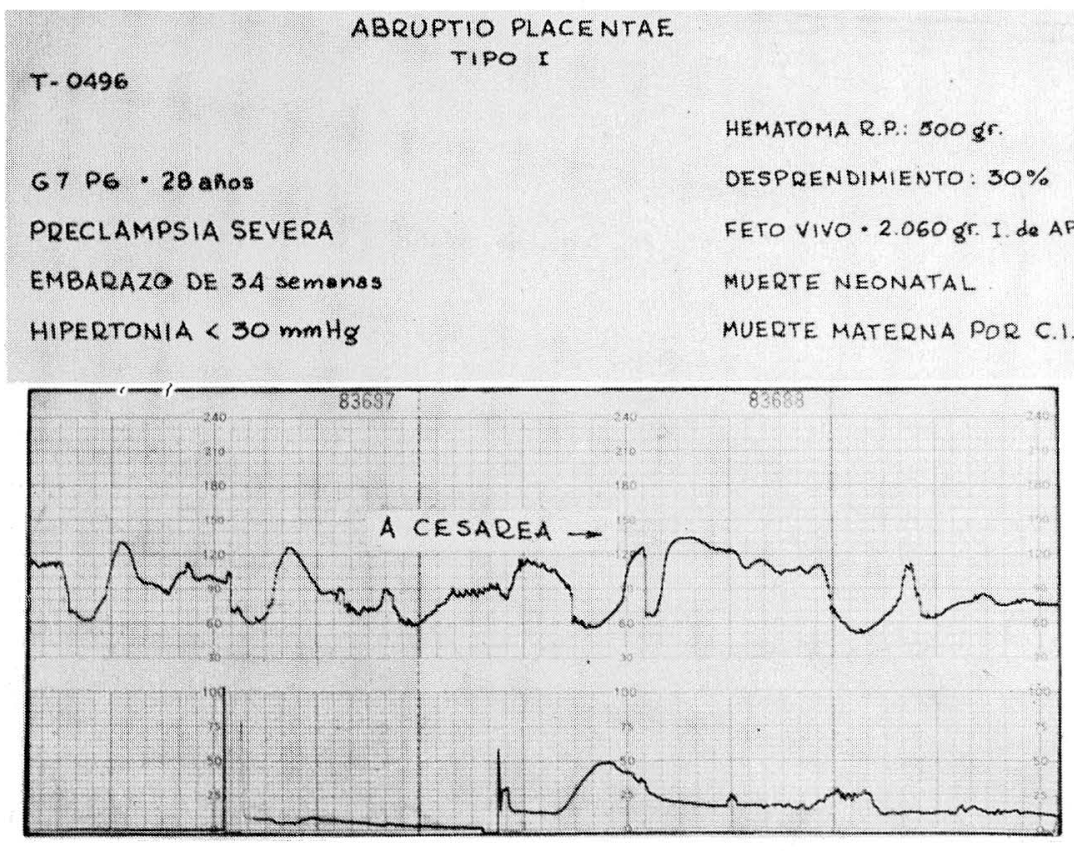

Fig. No. 11. Cambios periódicos combinado con alteración de la línea de la base. Nótese

ten una gradación de la severidad del compromiso fetal, como son la pérdida de variabilidad de la línea de base de la FCF o la presencia de un patrón sinusoidal o saltatorio persistente, los cuales se han descrito como signos que preceden a la muerte fetal $(11,12)$. Además la presencia de desaceleraciones variables con componente tardío y que a continuación presentan una disminución de variabilidad, hacen que el pronóstico sea más sombrío. Finalmente, si el cuadro anterior es seguido por una bradicardia - una desaceleración prolongada de 60 ó más latidos/minuto se debe considerar la posibilidad de un cuadro pre-morten (Figura 12 Panel A y B). Similarmente, se ha descrito este cuadro como precediendo a la muerte fetal "in útero" durante el período anteparto (14). el índice de Apgar $y$ el resultado final del Recién Nacido.

4. La insuficiencia úteroplacentaria que se hace manifiesta en entidades tales como toxemia temprana, enfermedad hipertensiva o cualquier otra causa de tipo crónico, pueden ser factores contribuyentes a la desnutrición intrauterina $y$ al desprendimiento placentario. Esta afirmación deberá ser explorada con mayor profundidad.

5. Debe tenerse en cuenta que no sólamente la muerte fetal o neonatal son importantes frente a la presencia de signos de anoxia intrauterina, sino que también debe pensarse en la evolución del recién nacido y su futuro desde el punto de vista neurolóyico. De allí la importancia de establecer un sistema adecuado de monitoría ante e intraparto, que permita diagnosticar precozmente la anoxia 

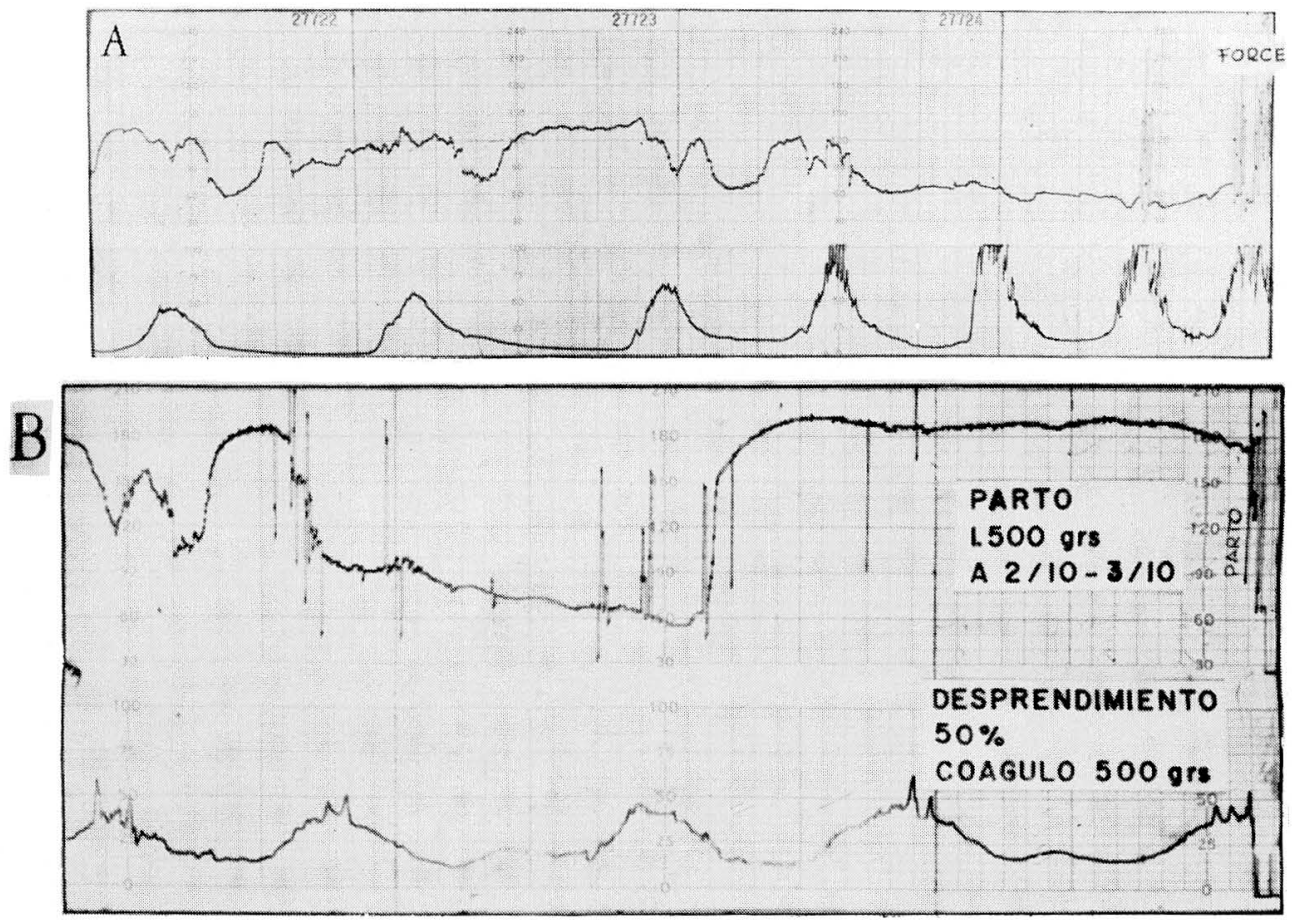

Fig. No. 12. Panel A y B. Panel A. Patrón de FCF que muestra alteraciones de la variabilidad, cambios periódicos y signos de empeoramiento que obligaron un parto intervenido. Panel B.

fetal y poder obrar rápidamente antes que esta agresión alcance una magnitud tal que cause un daño neurológico permanente, como se ha demostrado en primates con disminución experimental del aporte de oxígeno materno a la unidad feto placentaria (15), similar a lo que ocurría en el abruptio placentae.

\section{Recomendaciones}

1. La clasificación del grado y el tipo de abruptio debe hacerse con la máxima precisión posible, utilizando el criterio clínico y los métodos de monitorización disponibles o sea monitoría biofísica y bioquímica.
Idem del anterior con recuperación de la línea de la base. Observe los resultados del Recién Nacido.

2. La mayoría de los casos (aproximadamente el $70 \%$ ) son tributarios de operación cesárea, encontrándose la mayor proporción de estos casos en Abruptios de tipo II (hipertonía severa y actividad uterina elevada).

3. Frente a signos biofísicos de sufrimiento fetal como alteraciones de la línea de base (Pérdida de la Variabilidad, Bradicardia, Taquicardia, Ritmo Sinusoidal o Saltatorio) y cambios periódicos (desaceleraciones tardías o variables severas aisladas o en forma combinada), debe intentarse tratamiento médico tendiente a mejorar el estado materno fetal por un periodo de tiempo máximo de 
30 minutos, al término de los cuales se practicará la operación cesárea si no hay mejoría del cuadro; si se obtiene mejoría se pensará en el parto vaginal dentro de las máximas condiciones de vigilancia materna y fetal.

4. Se considera proscrita la administración de oxitocina en los casos de abruptio placenta con feto vivo, independientemente del tipo de Abruptio tipo I con feto muerto.

\section{Resumen}

Se hizo una revisión de los casos de desprendimiento prematuro de placenta normoinserta (Abruptio Placenta) presentados en el período comprendido entre el 1o. de Julio de 1975 y 30 de Junio de 1979, con el fin de analizar su manejo desde el punto de vista fetal, median- te monitoría electrónica y evaluar los cambios de la frecuencia cardíaca fetal (FCF), para tratar de establecer patrones de severidad entre estos casos.

Se analizaron dos grupos: aquellos que terminaron en operación cesárea $(58 \%)$ $y$ en parto vaginal $(42 \%)$ y teniendo en cuenta el grado o severidad de la hipertonía uterina, con el fin de establecer algunas normas tendientes a usar o no ocitocina y cuándo se debe esperar que evolucione el parto normal, siempre y cuando el patrón de compromiso fetal no contraindique este manejo conservador.

Se hacen los comentarios de acuerdo a los resultados obtenidos y se dan recomendaciones de tipo práctico para el manejo del abruptio placenta con feto vivo in útero.

\section{MONITORING OF FETAL CARDIAC FREQUENCY IN CASES OF ABRUPTIO PLACENTAE}

\section{Summary}

A revision was made of Abruptio Placentae cases detected in the period between july Ist 1975 and june 30, 1979. The purpose of this study was to analize fetal management through electronic monitoring and assessment of the changes in fetal cardiac frequency, so as to establish severity patterns in these cases.

Two groups were studied: those that concluded with Caesarian section $(58 \%)$,

\section{Referencias}

1. GOLDICH, I., BOYCE, E., J. A. M. A. 212, No. 2:288, 1970. Management of Abruptio Placentae. and those who had a normal delivery $(42 \%)$, considering the degree or severity of uterine hypertony. This was done in order to identify criteria for the use of oxitoxin, allowing some time for normal delivery to evolve as long as the fetus is not at risk.

Comments are made in accordance with the data obtained and practical recommendations are given for the management of Abruptio Placentae when the fetus is alive in utero. 
3. PAGE, E. IBSTET. Gynec. 3:385, 1954. Abruptio Placentae, dangers of delay in delivery.

4. COBO, E., QUINTERO, C., G. STRADA AM. J. Obstet. Gynec. 93:1151, 1965. Utering Behavior in Abruptio Placentae: I-Contraction patterns and their reactivity to oxytocin.

5. BURNETT, C. J. Obstet. Gynec. Brit. Comm. 80:120, 1973. The Management of Abruptio Placentae.

6. DEVAlerA, E. Am. J. Obstet. Gy. nec. 100:599, 1968. Abruptio Placentae.

7. CARTER, B. Obstet. Gynecol. 29:30, 1967. Premature Separation of the Nor mally Implanted Placenta.

8. BRYANT, R. Am. J. Obstet. Gynec. $81: 480,1961$. Cesarean Section in Cincinnati, ohio, 1950-1959.

9. HON, E. H. AND OUILLIGAN, E. J. Classification of FHR II. Comm. Med. 33:779, 1967.
10. MARTIN, C. B. AND SCHIFRIN, B. Prenatal fetal monitoring in Aladjem, $\mathrm{S}$. and Brown, A. K. editors: Perinatal In. tensive Care, Chap. 1. St. Louis 1976. The C. V. Mosby, Company

11. CURTIS, L. C. SCHIFRIN, B. Obstet. Gynec. 48: No. 5, May, 1977. Fetal Heart Rate Patterns Preceding Death in Utero

12. MODANLOU, H., FREEMAN, R., et, al. Obstet. Gynec. 49. No. 5, May, 1977. Sinusoidal FHR Pattern and Severe Fetal Anemia.

13. GABBE, S. et. al. Obstet. Gynec. 49, No. 2, Feb. 1977. FHR Response to acute Hemorrage.

14. ROCHARD F., SCHIFRIN, B., et al. Am. J. Obstet. Gynec. 126:699, 1976. Non-stressed Fetal Heart Rate in the Antepartum Period.

15. ADAMSON, K., MYERS, R. Am. J. Obstet. Gynec. 128:893, 1977. Late Decelerations and brain tolerance of the fetal monkey to intrapartum asphyxia. 